\title{
Hardware-in-the-loop Validation of the Grid Explicit Congestion Notification Mechanism for Primary Voltage Control in Active Distribution Networks
}

\author{
K. Christakou*, M. Pignati*, R. Rudnik*, S. Sarri*, J.-Y. Le Boudec* and M. Paolone* \\ * École Polytechnique Fédérale de Lausanne, CH-1015 Lausanne, Switzerland \\ konstantina.christakou@epfl.ch,marco.pignati@epfl.ch,roman.rudnik@epfl.ch,styliani.sarri@epfl.ch, \\ jean-yves.leboudec@epfl.ch, mario.paolone@epfl.ch
}

\begin{abstract}
The Grid Explicit Congestion Notification control mechanism (GECN) is a broadcast-based real-time demandresponse mechanism designed for primary voltage control in Active Distribution Networks (ADNs) [1,2]. An extensive set of off-line simulations has indicated that GECN is a promising candidate for deployment in the real field. However, prior to the actual deployment of the control mechanism, it is crucial to validate its performance when controlling a real grid. For this purpose we design and develop a dedicated experimental Hardware-in-the-Loop (HIL) test platform for the real-time validation of GECN. The HIL architecture consists of a Real-Time Simulator (RTS) where a real distribution feeder is modeled, together with controllable loads and the associated measurement infrastructure composed by virtual PMUs. These virtual metering devices stream data, via Ethernet, to a local Phasor Data Concentrator suitably coupled with a Discrete Kalman Filter State Estimator. The estimated network state is received by a GECN network controller. The control loop is closed by transmitting the computed broadcast control signals back to the network buses in the RTS using a micro-controller. By using this experimental setup we are able to (i) assess the performance of the whole control process in terms of voltage optimality and time latencies in a realistic setting and (ii) implement the GECN controllers into dedicated equipment that with the proper ruggedization can be readily deployed in the real field.
\end{abstract}

Index Terms-Active distribution networks primary voltage control, probabilistic load control, real-time demand-response, hardware-in-the-loop, phasor measurement unit, real-time state estimation.

\section{INTRODUCTION}

W ITHIN the context of Active Distribution Networks (ADNs), development of dedicated optimal control strategies is necessary to achieve specific operation objectives in order to avoid potential violations of operational constraints of voltage limits and line currents (e.g., [3]-[11]). In this direction, the introduction of new generation of advanced metering devices such as Phasor Measurement Units (PMUs) (e.g., $[12,13])$ and the development of sub-second Real-time State Estimation (RTSE) algorithms (e.g., [14]-[16]) present new opportunities and will, eventually, facilitate the definition and deployment of new control processes in ADNs.

However, prior to the actual deployment of a new control mechanism in a real network, it is crucial to validate its behavior and performance. The reason is that the assessment of a control process in the real field is practically impossible. First, the true system state is hidden, therefore it is not possible to quantify the control performances. Second, emergency situations such as contingencies or disturbances that might occur in the grid can result in unpredictable control actions. To overcome these limitations, the control process operation can be validated in laboratory contexts using Real-Time Simulators (RTSs) and associated Hardware-in-the-Loop (HIL) setups.

In this direction, the goal of this paper is the experimental validation of the Grid Explicit Congestion Notification mechanism (GECN), first proposed in $[1,2]$, via a dedicated HIL setup. GECN is a unified control mechanism that acts on a fast time-scale and provides grid ancillary services by controlling heterogeneous energy resources via low bit-rate broadcastcontrol signals. It avoids individual point-to-point communication from the Distribution Network Operator (DNO) to every controllable resource and relies on State Estimation (SE) for the feedback channel. In particular, GECN is composed of two elements: (i) a centralized network controller that observes the system state, computes optimal nodal power set-points and translates them into signals that are sent to the network, and (ii) local GECN resource-controllers that receive the signals and manage the controllable resources' response.

In this work, in order to perform the real-time (RT) assessment of GECN, we design a dedicated HIL test platform. This experimental HIL setup consists of the following elements:

- A RTS model composed of: (i) the electrical network, represented by a real distribution feeder [17], as well as the controllable resources and the GECN local controllers [1], and (ii) all the measurement infrastructure composed PMUs (i.e., [18]-[20]);

- A Phasor Data Concentrator (PDC), which receives the PMU data, decapsulates it and forwards it to a Discrete Kalman Filter (DKF)-based RTSE (e.g., [18]) and a GECN network controller that uses the estimated state to compute optimal power set-points for voltage control and the corresponding GECN broadcast control signals [1];

- A micro-controller that is suitably coupled with the GECN network controller and receives the computed broadcast control signals. In the micro-controller, the GECN signals are transformed to analog voltage signals and they are transmitted back to the network buses in the RTS via dedicated analog inputs.

Using the aforementioned HIL setup and realistic highfrequency sampled time-series for solar panels injections and consumption, first the operation of GECN is validated when 
applied to the case of thermostatically controlled loads for providing primary voltage control. Furthermore, to the best of our knowledge this is the first HIL platform where all the chain including the models of the network and measurement devices, the RTSE and the control mechanism is presented. This allows us to assess the performances of the whole process.

The structure of the paper is as follows. Section II gives the necessary background on primary voltage control via broadcast signals. The functionalities of the centralized GECN network controller and the design of the GECN load controller for the case of thermostatically controlled loads (TCLs) are briefly recalled. Section III focuses on the architecture of the HIL setup. In Section IV we validate the RT implementation of the GECN network controller and the load controller via regression testing. In Section $\mathrm{V}$ we assess the performance of GECN in RT by applying it to the case of a real ADN located in the Netherlands equipped with large populations of TCLs. Finally, Section VI concludes the paper with the final remarks and possible directions for future work.

\section{Summary of the GECN Control ArChitecture}

In this section, we describe briefly the main principles of the control mechanism we wish to validate, namely the "Grid Explicit Congestion Notification" (GECN), first proposed in [1]. GECN is conceived to provide primary voltage control by means of low bit-rate broadcast control signals sent to large populations of elastic loads connected to the network buses. The implementation of the control mechanism is based on the closed loop depicted in Fig. $1^{1}$.

In particular, first, a state estimator provides, at each time step, the state of the network in each bus $i$, i.e., nodal voltage phasors $\bar{E}_{i}(t)^{2}$. This information together with the knowledge of the network admittance matrix allows the DNO to compute also the nodal power injections, $P_{i}(t), Q_{i}(t)$. At the next time step, the goal of the DNO is to match as closely as possible a day-ahead scheduled consumption profile $\left(P_{i}^{f}(t+1), Q_{i}^{f}(t+\right.$ $1)$ ), while ensuring a voltage profile within acceptable operating limits $\left(E_{0}-\delta \leq\left|\bar{E}_{i}(t+1)\right| \leq E_{0}+\delta\right.$, where $\delta$ denotes the value of the voltage deviation from the rated value tolerated by the DNO). We consider that, in absence of control, the mismatch in bus $i$ is $\Delta P_{i}^{f}(t):=P_{i}^{f}(t+1)-P(t)$ and $\Delta Q_{i}^{f}(t):=Q_{i}^{f}(t+1)-Q(t)$. To this end, the DNO computes optimal required power adjustments $\left(\Delta \mathbf{P}^{*}(t), \Delta \mathbf{Q}^{*}(t)\right)$ for each bus $i$ that is equipped with controllable loads. In order to do so, first, the DNO computes voltage sensitivity coefficients

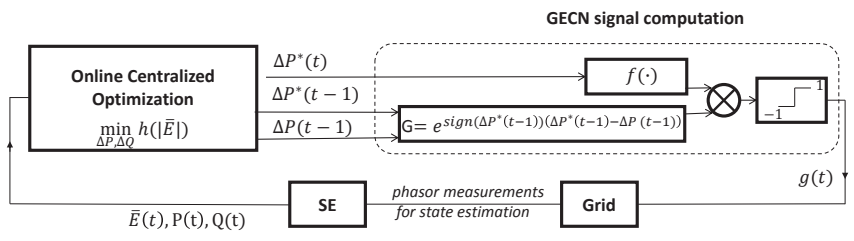

Fig. 1: Control loop for the computation of the GECN signal $\mathbf{g}(t)$ for the control of active power. Adapted from [1].

${ }^{1}$ Fig. 1 shows the closed loop for the control of the active power. A similar feedback control is adopted for the reactive power in case the controllable resources have reactive power capabilities.

${ }^{2}$ The rated value of the voltage in the network is denoted by $E_{0}$. with respect to absorbed/injected power of a network bus $\ell[1,21]$ :

$$
K_{P, i \ell}(t):=\frac{\partial\left|\bar{E}_{i}(t)\right|}{\partial P_{\ell}}, K_{Q, i \ell}(t):=\frac{\partial\left|\bar{E}_{i}(t)\right|}{\partial Q_{\ell}},
$$

This allows for a local linearization of the voltage deviation $\Delta|\overline{\mathbf{E}}(t)|=\left(\Delta\left|\bar{E}_{i}(t)\right|\right)_{i}$ :

$$
\Delta|\overline{\mathbf{E}}(t)| \approx \mathbf{K}_{\mathbf{P}}(t) \Delta \mathbf{P}(t)+\mathbf{K}_{\mathbf{Q}}(t) \Delta \mathbf{Q}(t) .
$$

Next, using the computed sensitivity coefficients, the DNO solves the following constrained optimization problem to compute nodal power adjustments which lead to the desired operating set-point for voltage control (online centralized optimization block shown in Fig. 1) ${ }^{3}$ :

$$
\min _{\Delta \mathbf{P}, \Delta \mathbf{Q}} \sum_{i}\left(\Delta P_{i}(t)-\Delta P_{i}^{f}(t)\right)^{2}+\sum_{i}\left(\Delta Q_{i}(t)-\Delta Q_{i}^{f}(t)\right)^{2}
$$

subject to:

$$
\begin{aligned}
& \gamma_{i} \leq \cos \varphi_{i} \leq 1 \\
& E_{\min } \leq\left|\bar{E}_{i}(t)\right|+\Delta\left|\bar{E}_{i}(t)\right|-E_{0} \leq E_{\max }
\end{aligned}
$$

where $\gamma_{i}$ is the constraint on the power factor of the $i$-th bus.

For the sake of reliable deployment of the computed setpoints the optimal control problem has been modified compared to [1]. In particular, the allowed limits on the voltage deviations are considered here explicitly as constraints of the optimization problem. Additionally, the penalty method is applied to transform the problem above into an unconstrained optimization problem and a gradient descent iterative scheme is used to compute the optimal solution of the control problem [22]. Adopting such a method allows us to have control over the solution of the optimization problem in real-time.

The resulting optimal set points, $\left(\Delta \mathbf{P}^{*}(t), \Delta \mathbf{Q}^{*}(t)\right)$ are mapped to a signal $g(t)=\left(g_{P, i}(t), g_{Q, i}(t)\right)_{i}$ with components in the range $[-1,1]$ corresponding to active and reactive power adjustments for each bus $i$. For both active and reactive power, a negative $g$ encourages consumption, a positive $g$ inhibits consumption, and $g=0$ does not affect the behavior of the controllable resources. At time $t, g_{p}(t)$ is a function of (i) the optimal set points at the current time-step and (ii) the mismatch $G$ between the optimal and the actual set points that the DNO observed at the previous time step $t-1$ (GECN signal computation block in Fig. 1).

Next, the computed GECN signals are broadcasted to the network buses. The various distributed resources in network bus $i$ receive a single broadcast signal $\left(g_{P, i}, g_{Q, i}\right)$. The local controller of a certain resource attached to this bus decides the action to be taken based on the internal state of the resource and on the value of the received signal. In the rest of the paper we assume that large populations of controllable TCLs are present in the network buses, modeled in our case as refrigerators. In this case, the appliances operate normally in an $\mathrm{ON}-\mathrm{OFF}$ mode in a temperature deadband absorbing active power when on and, at the same time, a proportional amount of reactive power via a fixed power factor value. Therefore the GECN local controller targets only the control of active power injections by requesting the TCLs to switch mode

\footnotetext{
${ }^{3}$ The objective function in 3 corresponds to function $h$ in Fig. 1.
} 


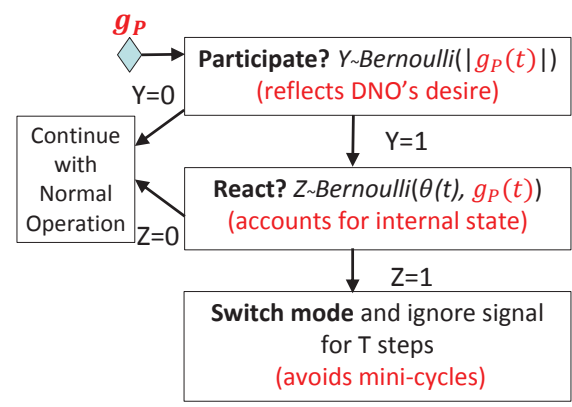

Fig. 2: State model representing the local controllers for TCLs.

when necessary. The design and actions of the GECN local resources-controller are shown in the flow chart of Fig. $2^{4}$. In particular, a fridge controller reacts to a $g_{P}$ signal with a probability equal to the signal magnitude $\left(\left|g_{P}(t)\right|\right)$. In this way, the larger the signal the more appliances will participate in the control action. Next, if this first test is passed, the controller takes the decision to turn on or off the appliance with a certain probability that depends on the signal and on the internal state of the fridge, i.e., its internal temperature $(\theta(t), g(t))$. Finally, if an appliance switches mode due to a GECN signal then it ignores all subsequent control signals for a predetermined number of time steps. This ensures that operation in mini-cycles is avoided in order to preserve the compressor's lifetime.

Finally, the resulting variation of the aggregate power at the buses provides the DNO with an implicit feedback with respect to the responsiveness of the bus resources. This variation plays a role in deciding the control actions for subsequent time steps.

\section{HIL SETUP AND EXPERIMENT DESIGN}

The HIL setup that has been designed and implemented for the experimental validation of the GECN control scheme is shown in Fig. 3. It consists of a RTS that communicates via the local Ethernet network with a workstation. In particular, we use the Opal-RT eMEGAsim PowerGrid Real-Time Digital Simulator equipped with a Spectracom Tsync-PCIe express GPS synchronization module ([23]). In the RTS, we have developed specific models to represent the electrical network, the PMUs, the TCLs and the GECN load controllers. In addition to the controllable resources, non-dispatchable production coming from distributed solar panel units and non-controllable demand are also included in the RTS.

In the RTS, three-phase bus voltage and injected current signals, i.e., the true system state, are forwarded to the PMUs that are installed in some of the network buses. It is worth noting that the developed set-up corresponds to a real grid and the location of the PMUs corresponds to the real installation of these devices in the ADN. This location depends on installation constraints set by the DNO, as well as on the network observability. The PMUs estimate the synchrophasors of nodal voltages and injected/absorbed currents, encapsulate them according to the IEEE Std. C37.118.2-2011 and stream the relevant frames via Ethernet to the workstation. More details on the model of the RTS PMU can be found in [19].

In the workstation, a specific LabVIEW interface comprises a PDC, a RTSE, as well as the GECN network controller.

\footnotetext{
${ }^{4} \mathrm{~A}$ more detailed description of this load controller is given in [1].
}

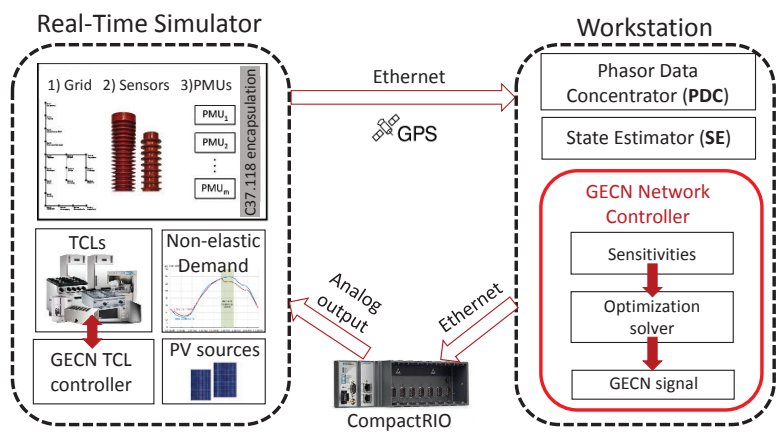

Fig. 3: The proposed HIL setup for the validation of GECN.

The dataframes streamed by the PMUs are received first by the PDC where they are decapsulated, aggregated and timealigned. Next, they are passed to a Kalman Filter (KF)-based SE. A detailed description of both the PDC and the RTSE process can be found in $[18,24]$. In this work we rely on a PMU-based RTSE. The reason is that the control needs of ADNs, that require in principle the knowledge of the system state, span time windows that range from few hundreds of milliseconds (e.g., fault management) to a few seconds (e.g., voltage control and line congestions management). PMUs are compatible with the stringent time requirements as they can stream data in the order of few tens of synchrophasors per second and, therefore, allow the development of sub-second SE processes. However, the control approach described in this paper can be extended to the case of SE based on Remote Terminal Units (RTUs) (i.e., relying on measurements coming from smart meters).

Once the estimated state is available, it is received by the GECN network controller, implemented also in LabVIEW, that uses it to compute the broadcast control signals. To this end, first the voltage sensitivity coefficients are computed ([21]) and next, the optimal control problem in 3 is solved. Finally, the GECN broadcast control signal is computed. The operation of the GECN network controller is triggered every $16 \mathrm{sec}$ as in [1]. In order to close the control loop, the GECN signal is sent via Ethernet to a micro-controller where it is transformed to an analog voltage signal and transmitted via dedicated analog outputs back to the network buses in the RTS. There, each signal is received by all the GECN load controllers that are connected to a single network bus. The local controllers change the state of the TCLs according to the received signal and, consequently, the network state.

The ADN model in the RTS is the BML 2.10 feeder, namely a real 18-buses medium voltage feeder, part of the Alliander electrical distribution grid located in the Netherlands 5 . Its topology is shown in Fig. 4(a). The voltage of the slack bus (bus 1) is equal to $10 \mathrm{kV}$ RMS line-to-line. The network short-circuit power is equal to $300 \mathrm{MVA}$ and $R_{c c} / X_{c c}=0.1$. Fig. 4(b) shows the physical area where the feeder of interest is located, as well as the PMU installation. Ten out of the network buses are equipped with PMUs. In particular buses 1, 3, 5, 6, 8, 10, 12, 13, 15 and 17 in Fig. 4(a) that correspond to the blue squares locations shown in Fig. 4(b). At each of the buses equipped with PMUs, apart from bus 1, a population of 400 controllable refrigerators is considered. The characteristics

\footnotetext{
${ }^{5}$ The data of this feeder have been made available by Alliander within the context of the C-DAX FP7 EU project (http://www.cdax.eu/).
} 


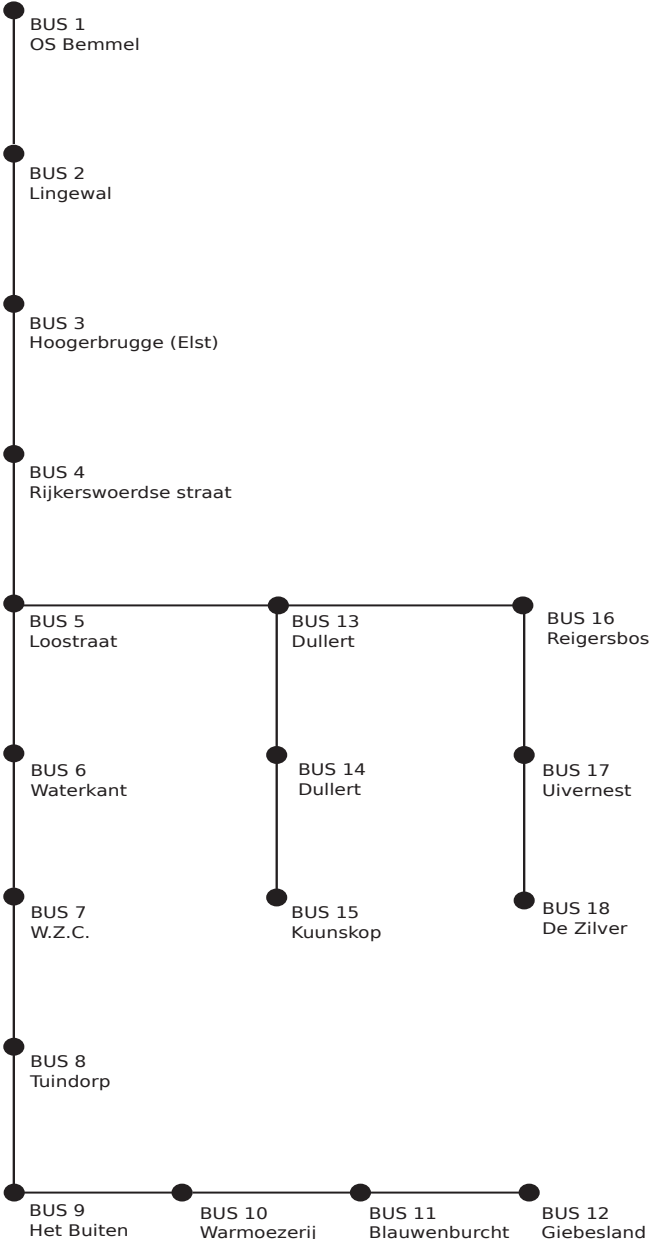

(a) The Alliander $10 \mathrm{kV}$ feeder BML 2.10 .

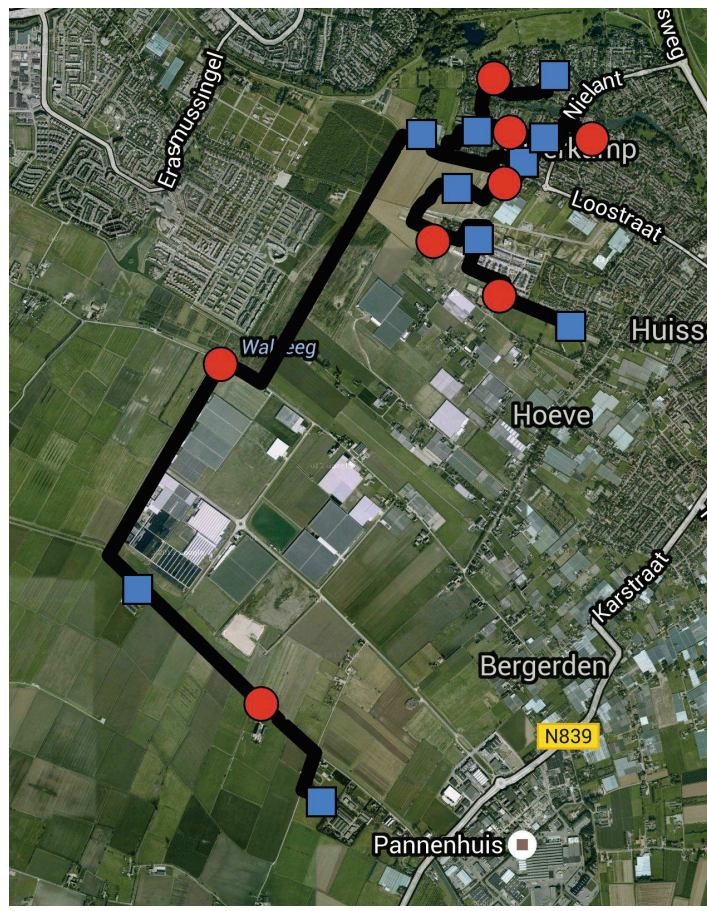

(b) Location of PMUs in the BML 2.10 feeder (blue squares).

Fig. 4: Topology and location of PMUs in the Alliander BML 2.10 feeder.
TABLE I: Parameters of the elastic appliances and the load controller

\begin{tabular}{|c|c|}
\hline Parameter & Value \\
\hline Temperature deadband, $\Theta\left({ }^{\circ} \mathrm{C}\right)$ & {$[1,6]$} \\
\hline Ambient temperature, $\theta_{0}\left({ }^{\circ} \mathrm{C}\right)$ & 19 \\
\hline Thermal conductivity, $\mathrm{A}\left(\mathrm{kW} /{ }^{\circ} \mathrm{C}\right)$ & 10.563 \\
\hline Coefficient of performance, $(\eta)$ & 3 \\
\hline Rated power, $P_{r}($ Watt $)$ & 150 \\
\hline Time step, $\tau(\mathrm{sec})$ & 1 \\
\hline Time constant, $T_{c}=m_{c} / A(\mathrm{hrs})$ & $\sim U(1.326,2.778)$ \\
\hline Controller time counter, $T_{0}(\mathrm{sec})$ & 480 \\
\hline Internal state parameter, $\xi$ & 0.4 \\
\hline Appliance power factor, $\cos \varphi$ & 0.85 \\
\hline
\end{tabular}

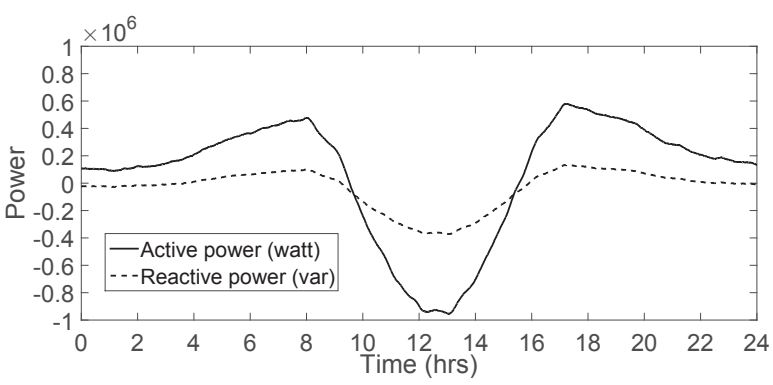

Fig. 5: Aggregated non-controllable active and reactive power injections of all network buses.

of the elastic appliances, as well as the TCL controller parameters are shown in Table I. In addition to the controllable resources, real measurements of $24 \mathrm{hr}$ curves of consumption and production coming from distributed photovoltaic (PV) units are considered in each network bus. The aggregate active and reactive power injections profiles of all the network buses are shown in Fig. $5^{6}$.

At this point, it is worth noting that the proposed design of the HIL setup is not limited to the validation of the GECN control mechanism. In particular, the RTS model of the measurement infrastructure, as well as the PDC and the RTSE modules of the HIL setup can be deployed for the validation of different control applications according to the DNO's requirements. In what follows, we first verify that the RT implementation of GECN is the same as the off-line eventdriven simulation in Matlab by performing a regression testing. Then the performance of GECN is evaluated in terms of voltage optimality and its time requirements are characterized using the network and power profiles presented in this section.

\section{Regression Testing}

In this section we want to verify that the implementation of the GECN control mechanism in real-time is identical to the off-line version in Matlab used for the simulations in [1]. For this purpose, the goal is to run the same test-case in Matlab off-line and in real-time and obtain identical resulting voltage profiles, as well as aggregated power of the controllable TCLs. However, there are several factors that render such a comparison difficult. In particular, the probabilistic nature of the GECN local controllers and the noise introduced by the analog signals do not allow to obtain a deterministic output of the controllable loads between different simulations. Furthermore, the finite integration time-step of the RTS involves truncation

${ }^{6}$ These aggregate data come from measurements taken at a different feeder other than the BML 2.10. 
errors caused by the RTS solver that are different from the event-driven simulator developed in Matlab that, instead uses the power flow calculus. For all these reasons, we choose to test and compare separately the operations of the GECN network controller and the load controllers as described next.

For the validation of the GECN network controller, the following test is performed. We first run a $24 \mathrm{hr}$ off-line Matlab simulation and we store every second the network state, i.e., the nodal voltage phasors, the nodal power injections, as well as the computed GECN signals for each network bus. Then, we use the nodal voltage phasors and power injections as input to the LabVIEW implementation of the GECN network controller block shown in Fig. 3. In this way, we can compare the $24 \mathrm{hr}$ GECN signals computed in Matlab with the ones obtained from the GECN network controller that is used in the experimental set-up. The results of this test are shown in Fig. 6- 7. In these figures the difference between the 24hr GECN signal computed off-line in Matlab and the one computed by the RT implementation in LabVIEW is shown. For the sake of brevity only the signals sent to bus 3 (Hoogerbrugge-Elst), which is the controllable bus closest to the slack bus and bus 12 (Giebesland) which is the furthest one are shown. As it can be observed, the difference between the GECN signals is negligible, in the order of $10^{-11}$, which indicates that the implemented GECN network controller is behaving in RT as the event-driven one developed in Matlab.

For the validation of the local controllers of the TCLs a similar procedure is adopted. We run a $24 \mathrm{hr}$ off-line simulation in Matlab and we store the GECN signals sent to the medium voltage (MV) network buses, as well as the aggregate power of the controllable resources. Then we use the control signals as input to the RT TCL controller which is implemented in Simulink and we observe the aggregate TCL power at each MV bus. In both the RT and the off-line simulations we make sure to set the seed of the random number generator so that

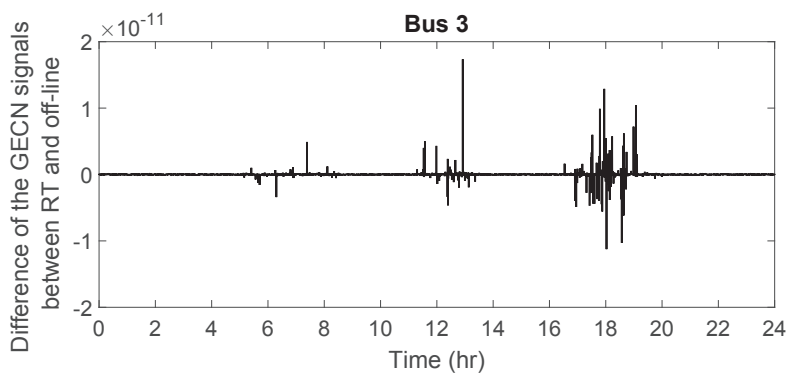

Fig. 6: Difference between the $24 \mathrm{hr}$ GECN signals sent to bus 3 computed by the Matlab off-line and the LabVIEW RT implementation of the GECN network controller.

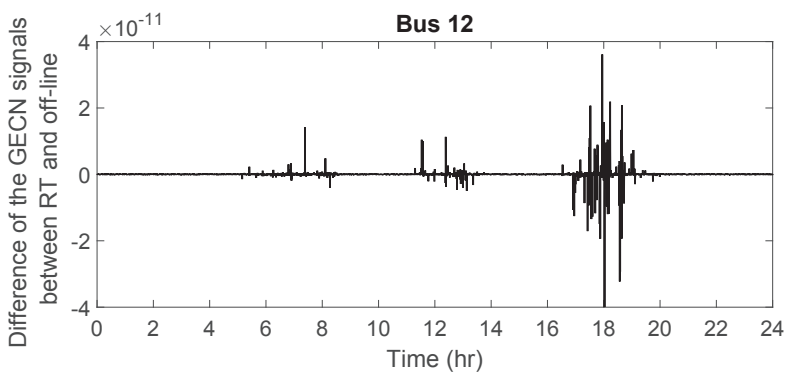

Fig. 7: Difference between the $24 \mathrm{hr}$ GECN signals sent to bus 12 computed by the Matlab off-line and the LabVIEW RT implementation of the GECN network controller.

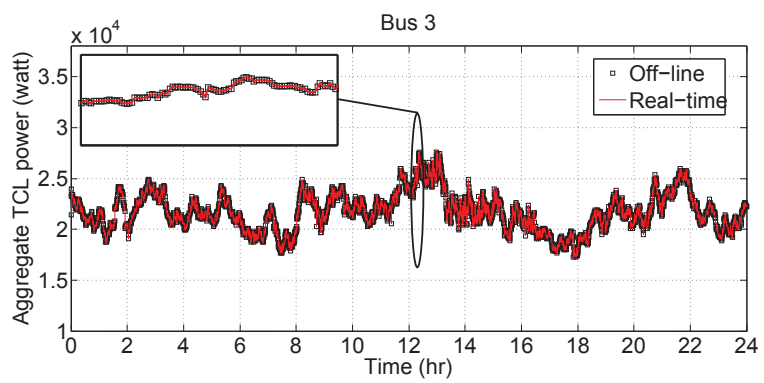

Fig. 8: Aggregate power consumption of TCLs connected to bus 3 in Matlab off-line (black squares) and in RT simulation (red line).

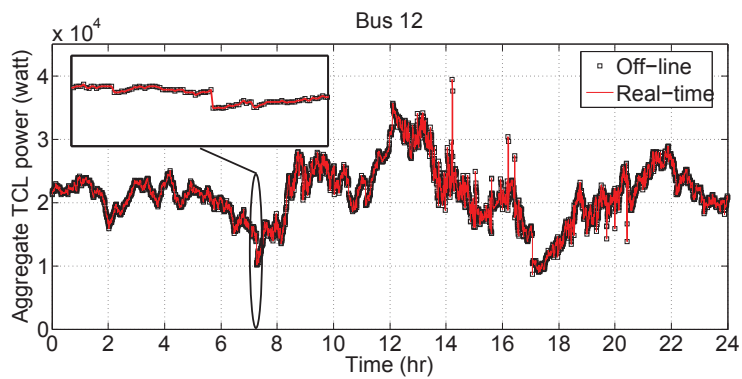

Fig. 9: Aggregate power consumption of TCLs connected to bus 12 in Matlab off-line (black squares) and in RT simulation (red line).

the same sequence of random numbers is produced in the two simulations. By doing so we are able to compare the two different implementations of the local resources controller in the two different software platforms. Fig. 8 and 9 show the aggregate TCL power of bus 3 and 12 respectively computed in the off-line simulations (black squares) and in real-time (red line $)^{7}$. As it can be observed in these figures the aggregate powers of the TCLs are exactly superposed, indicating that the TCL controller, as well as the TCL model are behaving in RT exactly as the ones in Matlab.

\section{Demonstration Example and Performance ASSESSMENT}

In this section the performances of GECN as a RT primary voltage controller in ADNs are evaluated. To this end we use the real distribution feeder shown if Fig. 4 and the HIL set-up described in detail in Section III.

First, we run a $24 \mathrm{hr}$ RT simulation without enabling the GECN network controller in order to obtain the base-case voltage profile, as well as the uncontrolled aggregate consumption of the TCLs. The results of this simulation for the voltage are shown in the dashed gray lines in Fig. 10(a)-11(a). For the sake of brevity, only the network voltage profiles that exhibit the minimum and maximum voltage variations are shown, namely the one of bus 3 and bus 12 that are the closest and the furthest away from the slack bus respectively. It is worth observing that for bus 12, the absence of a suitable control produces voltages below the allowed limit of 0.95p.u. in correspondence of the peak consumption periods, i.e., hours 7-8 and 17-18. Moreover, over-voltages above 1.05p.u. occur in the middle of the day during the peak production of the PV units, i.e. hours 12-13. In Fig. 10(c)-11(c) the aggregate power of the

\footnotetext{
${ }^{7}$ Note that in this case the differences between the aggregate powers computed in the two implementations are not shown as they are exactly zero for the whole $24 \mathrm{hr}$ period.
} 


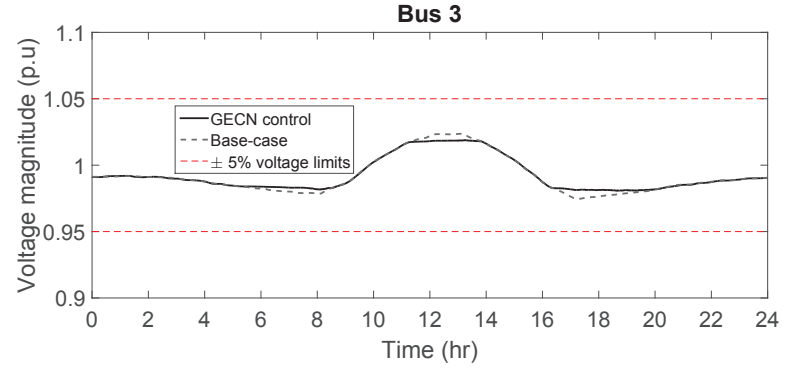

(a) $24 \mathrm{hr}$ voltage profile of bus 3 before (gray dashed line) and after (black line) the GECN control.

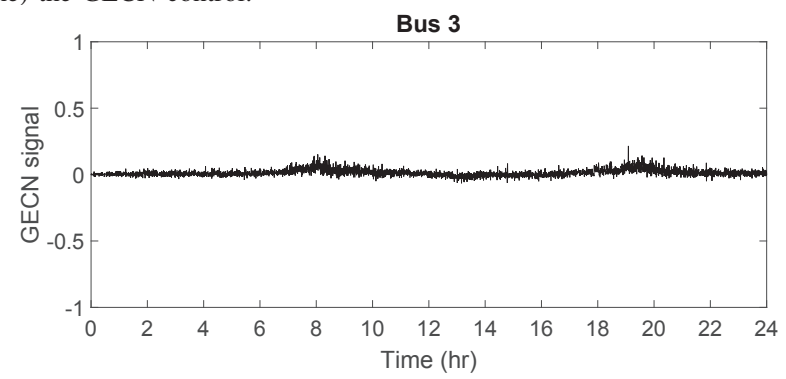

(b) $24 \mathrm{hr}$ GECN signal sent to bus 3 .

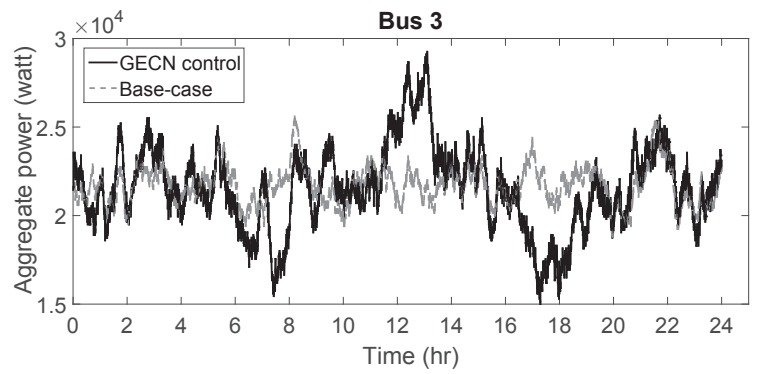

(c) $24 \mathrm{hr}$ aggregate power consumption of the TCLs connected to bus 3 before (gray curve) and after (black curve) the GECN control.

Fig. 10: Voltage profile, GECN signal and aggregate TCL power of bus 3 .

refrigerators in buses 3 and 12 respectively are shown for the base-case in gray.

Next, we run a $24 \mathrm{hr}$ RT simulation where GECN control is enabled. The improvement in the voltage profile of buses 3 and 12 due to the application of GECN is shown in Fig. 10(a)11(a) (black curves). It is worth noting that the GECN is able to control in real-time the network voltage guaranteeing that the resulting profiles remain for the whole $24 \mathrm{hr}$ period within the allowed $\pm 5 \%$ limits shown in the dashed red lines. The GECN signals that correspond to this improvement in the voltage profiles are shown in Fig. 10(b)-11(b) for bus 3 and 12 respectively. As expected, the signal sent to bus 12 exhibits larger magnitudes caused by the larger voltage variations in this bus, whilst GECN signals are close to zero for bus 3. In fact, the three peaks of the signal observed in Fig. 11(b) correspond to the time periods when undervoltages and over-voltages occur in Fig. 11(a), i.e., hours 7-8, 12-13 and 17-18. The GECN signals cause variations of the aggregate TCL power of the controllable buses that can be observed in Fig. 10(c)-11(c) in black. Compared to the base-case consumption (gray curves) the TCLs consume less during hours 7-8 and 17-18, responding correctly to the positive GECN signal that dictates there is a peak in consumption that causes under-voltages. On the contrary, the TCLs consume more during hours 12-13 in order to locally

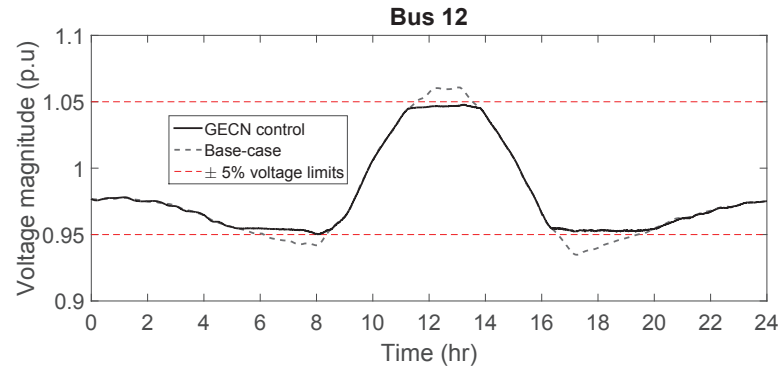

(a) $24 \mathrm{hr}$ voltage profile of bus 12 before (gray dashed line) and after (black line) the GECN control.

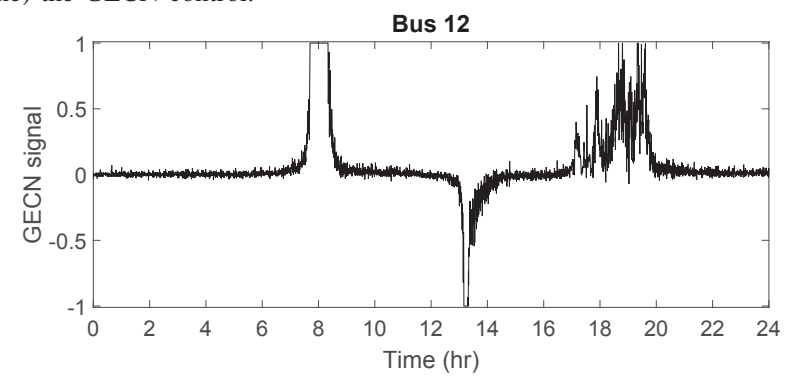

(b) $24 \mathrm{hr}$ GECN signal sent to bus 12 .

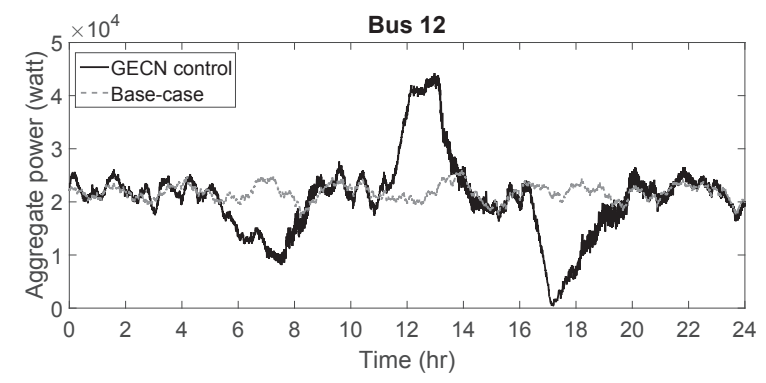

(c) $24 \mathrm{hr}$ aggregate power consumption of the TCLs connected to bus 12 before (gray curve) and after (black curve) the GECN control.

Fig. 11: Voltage profile, GECN signal and aggregate TCL power of bus 12 .

compensate the peak in the PV power production and decrease the corresponding over-voltages. Overall, these results indicate that the GECN control mechanism is able to selectively control the aggregated demand per bus and successfully provide realtime primary voltage control in active distribution networks.

In addition to the performance evaluation of GECN in terms of voltage optimality, it is interesting to assess the time latencies of the control process. In Fig. 12 the time required by the GECN network controller to solve the centralized optimization problem throughout the $24 \mathrm{hr}$ period is shown. It is worth noting that even during the time-periods when voltage control is required in the network, the solution time of the optimization problem is in the order of few milliseconds. Furthermore, Fig. 13 shows the CDFs of the time required to solve the centralized optimization problem off-line, using the solver fmincon of Matlab and in RT, using the gradient descent method. It is worth noting the significant improvement of the adopted solution method in RT which is in the order of ten times faster. In particular, the median value of the solution time is $1.12 \mathrm{~ms}$ in RT with a corresponding 95 -th percentile of $2.70 \mathrm{~ms}$, where as off-line these values are $17.37 \mathrm{~ms}$ and $32.82 \mathrm{~ms}$ respectively. In [18] the authors characterize the latency of the whole process from the moment the data is arrived to the PDC until the state is available from the RTSE for the case of the BML 2.10 feeder and they find that this 


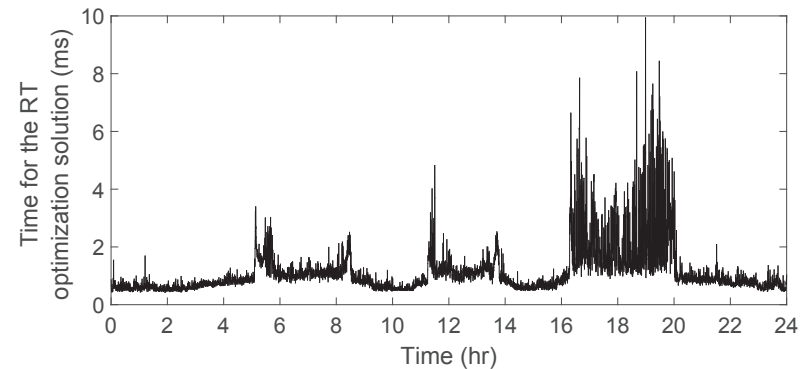

Fig. 12: Time required for the solution of the optimization problem.

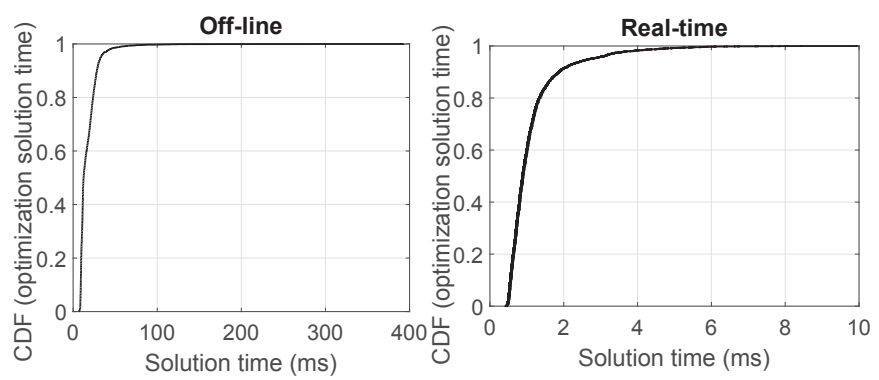

Fig. 13: CDF of the time required to solve the optimal control problem, comparison between off-line and RT implementations.

latency is in the order of $20 \mathrm{~ms}$. Therefore, taking into account the time latencies shown in Fig 12, within roughly $35 \mathrm{~ms}$ from the moment the data is available to the PDC we are able to solve the centralized optimization problem and compute the GECN signals. Overall, the timing performance shown here confirms the adequateness of GECN as a primary controller.

\section{CONCLUSiOnS}

In this paper we have validated experimentally the operation of GECN, a primary voltage control mechanism for ADNs. For this purpose, we have designed a specific HIL set-up that allowed us to evaluate in RT the performance of GECN in terms of voltage optimality and to characterize the time latency of the control process. The validation has been done using a real medium voltage feeder located in the Netherlands, part of the Alliander $10 \mathrm{kV}$ electrical distribution grid. The RT experimental validation has shown that GECN can successfully maintain the network voltage profile within the acceptable limits for safe operation (typically $\pm 5 \%$ of the network rated value). Furthermore, we have computed the time required for the solution of the optimal control problem by the centralized GECN network controller and we have shown that it is in the order of few ms. Such time requirements indicate the adequateness of GECN as a primary voltage control scheme. Finally, the RT implementation of the GECN network controller, as well as TCL controllers into dedicated equipment can, in principle, facilitate the actual deployment of the control process in the real field.

\section{REFERENCES}

[1] K. Christakou, D.-C. Tomozei, J.-Y. Le Boudec, and M. Paolone, "Gecn: Primary voltage control for active distribution networks via real-time demand-response," IEEE Trans. on Smart Grid, vol. 5, no. 2, pp. 622631, March 2014

[2] K. Christakou, D.-C. Tomozei, M. Bahramipanah, J.-Y. Le Boudec, and M. Paolone, "Primary voltage control in active distribution networks via broadcast signals: The case of distributed storage," IEEE Trans. on Smart Grid, vol. 5, no. 5, pp. 2314-2325, Sept 2014.
[3] N. Singh, E. Kliokys, H. Feldmann, R. Kussel, R. Chrustowski, and C. Joborowicz, "Power system modelling and analysis in a mixed energy management and distribution management system," IEEE Trans. Power Systems, vol. 13, no. 3, pp. 1143-1149, 1998.

[4] N. Jenkins, R. Allan, P. Crossley, D. Kirschen, and G. Strbac, Embedded generation, A. T. Johns and D. F. Warne, Eds. IET, 2000, vol. 9, no. 3.

[5] N.-G. James, Control and Automation of Electrical Power Systems. Hoboken, NJ: CRC Press, 2006.

[6] A. Borghetti, M. Bosetti, S. Grillo, S. Massucco, C. Nucci, M. Paolone, and F. Silvestro, "Short-term scheduling and control of active distribution systems with high penetration of renewable resources," IEEE Systems Journal, vol. 4, no. 3, pp. 313-322, 2010.

[7] Q. Zhou and J. Bialek, "Generation curtailment to manage voltage constraints in distribution networks," IET Generation, Transmission \& Distribution, vol. 1, no. 3, pp. 492-498, 2007.

[8] T. Senjyu, Y. Miyazato, A. Yona, N. Urasaki, and T. Funabashi, "Optimal distribution voltage control and coordination with distributed generation," IEEE Trans. Power Delivery, vol. 23, no. 2, pp. 1236-1242, 2008.

[9] J. Lopes, N. Hatziargyriou, J. Mutale, P. Djapic, and N. Jenkins, "Integrating distributed generation into electric power systems: A review of drivers, challenges and opportunities," Electric Power Systems Research, vol. 77, no. 9, pp. 1189-1203, 2007.

[10] M. Delfanti, M. Merlo, G. Monfredini, V. Olivieri, M. Pozzi, and A. Silvestri, "Hosting dispersed generation on italian mv networks: Towards smart grids," in 2010 14th International Conference on Harmonics and Quality of Power (ICHQP), Sept 2010, pp. 1-6.

[11] E. Coster, J. Myrzik, B. Kruimer, and W. Kling, "Integration issues of distributed generation in distribution grids," Proceedings of the IEEE, vol. 99, no. 1, pp. 28-39, Jan 2011.

[12] P. Romano and M. Paolone, "Enhanced interpolated-dft for synchrophasor estimation in fpgas: Theory, implementation, and validation of a pmu prototype," IEEE Trans. on Instrumentation and Measurement, vol. 63, no. 12, pp. 2824-2836, Dec 2014.

[13] A. G. Phadke and J. S. Thorp, Synchronized phasor measurements and their applications. Springer Science \& Business Media, 2008.

[14] M. Pignati, M. Popovic, S. Barreto, R. Cherkaoui, G. Dario Flores, J.Y. Le Boudec, M. Mohiuddin, M. Paolone, P. Romano, S. Sarri et al., "Real-time state estimation of the epfl-campus medium-voltage grid by using PMUs," in Innovative Smart Grid Technologies Conference (ISGT), 2015 IEEE Power \& Energy Society. IEEE, 2015, pp. 1-5.

[15] J. Liu, J. Tang, F. Ponci, A. Monti, C. Muscas, and P. Pegoraro, "Tradeoffs in pmu deployment for state estimation in active distribution grids," IEEE Trans. on Smart Grid, vol. 3, no. 2, pp. 915-924, June 2012.

[16] A. von Meier, D. Culler, A. McEachern, and R. Arghandeh, "Microsynchrophasors for distribution systems," in IEEE 5th Innovative Smart Grid Technologies Conference, Washington, DC, 2014.

[17] W. K. Chai, N. Wang, K. V. Katsaros, G. Kamel, G. Pavlou, S. Melis, M. Hoefling, B. Vieira, P. Romano, S. Sarri et al., "An informationcentric communication infrastructure for real-time state estimation of active distribution networks," IEEE Trans. on Smart Grid, vol. 6, no. 4 pp. 2134-2146, 2015.

[18] S. Sarri, M. Pignati, P. Romano, L. Zanni, and M. Paolone, "A hardwarein-the-loop test platform for the performance assessment of a PMUbased real-time state estimator for active distribution networks," in PowerTech, 2015 IEEE Eindhoven, June 2015, pp. 1-6.

[19] P. Romano, M. Pignati, and M. Paolone, "Integration of an ieee std. c37. 118 compliant pmu into a real-time simulator," in PowerTech, 2015 IEEE Eindhoven. IEEE, 2015, pp. 1-6.

[20] M. Paolone, M. Pignati, P. Romano, S. Sarri, L. Zanni, and R. Cherkaoui, "A hardware-in-the-loop test platform for the real-time state estimation of active distribution networks using phasor measurement units," in Proc. Cigré SC6 Colloquium, 2013.

[21] K. Christakou, J. LeBoudec, M. Paolone, and D.-C. Tomozei, "Efficient computation of sensitivity coefficients of node voltages and line currents in unbalanced radial electrical distribution networks," IEEE Trans. Smart Grid, vol. 4, no. 2, pp. 741-750, 2013.

[22] D. P. Bertsekas, "Nonlinear programming," 1999.

[23] "Opal-RT eMEGAsim PowerGrid Real-Time Digital Hardware in the Loop Simulator [Online]." http://www.opal-rt.com/product/ emegasim-powergrid-real-time-digital-hardware-in-the-loop-simulator/.

[24] L. Zanni, S. Sarri, M. Pignati, R. Cherkaoui, and M. Paolone, "Probabilistic assessment of the process-noise covariance matrix of discrete kalman filter state estimation of active distribution networks," in International Conference on Probabilistic Methods Applied to Power Systems (PMAPS). IEEE, 2014, pp. 1-6. 